\title{
Regularization method for solving dual series equations involving heat equation with mixed boundary conditions
}

\author{
Naser A. Hoshan \\ Department of Mathematics, Tafila Technical University, Tafila, Jordan
}

Received: 1 March 2018, Accepted: 20 March 2019

Published online: 27 May 2019.

\begin{abstract}
In this article, the solution of a non-stationary heat equation in an axial symmetry cylindrical coordinates is determined, where the heat equation is being subject to non-homogeneous mixed discontinuous boundary conditions of first and second kind. In fact, the problem is transformed to a Fredholm integral equation of first kind, therefore the solution of the heat problem is determined by solving the Fredholm integral equation, where we use the regularization method to have the solution. In fact, the Laplace transform, Hankel transform and separation of variables are used for the problem transformation into the integral equation.
\end{abstract}

Keywords: Dual series equations, mixed conditions, regularization method.

\section{Introduction}

Because of the application of the mixed equations in physical problems, which are based on the time independent and Helmholtz equation, several articles have been studied the solution of mixed problem which has a dual equations $[9,6,4,3$, $5,8,7],[16],[15]$. For instance, in this article, we consider a mixed problem of solid cylindrical with an infinite height and given radius $R$. In particular, inside the disk $0<r<r_{0}$, the boundary conditions on the surface of cylindrical and outside the disk $r_{0}<r<R$ are given, and of first kind and second kind respectively, or vis versa. Our method for having the solution of heat equation with mixed boundary problem is summarized as follows. Firstly, we use the Laplace transform to the heat equation and to the mixed boundary conditions, then we use the separation of variables of the heat equation in axial cylindrical coordinates, where the problem is reduced to a dual series equations (DSE) with first kind Bessel function and order zero, such that the weight and the free terms depend on the parameter of the Laplace transform. Secondly, we use the inverse of Hankel transform to reduce the DSE into a first kind Fredholm integral equation. Finally, we use the regularization method for solving the resulting integral equation, where the regularization method based on converting the first kind integral equation into a second kind integral equation, such that several methods could be used for solving the integral equation numerically.

\section{Formulation and Solution of the Problem}

We consider the solution of the heat

$$
\theta_{r r}(r, z, \tau)+\theta_{r}(r, z, \tau) / r+\theta_{z z}(r, z, \tau)=\theta_{\tau}(r, z, \tau) / a
$$

With the mixed discontinuous boundary conditions of first and second kind of the form

$$
\theta_{z}(r, 0, \tau)=f_{1}(r, \tau) ; \quad 0 \leq r<r_{0}
$$




$$
\theta(r, 0, \tau)=f_{2}(r, \tau) ; \quad r_{0}<r<R
$$

where $\theta(r, z, \tau)=T(r, z, \tau)-T_{0}$ is the temperature distribution function, such that $T_{0}$ and $a$ are the given initial temperature constant heat diffusivity coefficient respectively, and $f_{i}(r, z) ; i=1,2$ are known continuous functions with respect to the variables $r, \tau$ which are accept with Laplace and Hankel transforms with respect to the variables $r$ and $\tau$ respectively. In fact, equation (1) is given over the surface of the cylinder $z=0$ and inside the disk $0<r<r_{0}<R$. On a surface $r=R$ and on the axis $r=0 \quad z \rightarrow \infty$, the unmixed homogeneous boundary conditions given as:

$$
\begin{array}{ll}
\theta(R, z, \tau)=0 ; & 0 \leq z<\infty \\
\theta(0, z, \tau)=0 ; & 0 \leq z<\infty \\
\theta(r, \infty, \tau)=0 ; & 0 \leq r<R
\end{array}
$$

In fact, the physical significance of the problem formulated such that, on the level surface of semi-infinite cylinder inside the disk $z=0 ; 0<r<r_{0}$, a mixed boundary condition of the second kind prescribed heat flux according Fourier low of heating, whereas outside the disk $z=0 ; r_{0}<r<R$, a temperature function is given. In the following, we will transform the problem to a Fredholm integral equation. applying the Laplace transform to equations (1) to (6), where

$$
\bar{\theta}(r, z, s)=L[\theta(r, z, \tau)]=\int_{0}^{\infty} \theta(r, z, \tau) \exp (-s \tau) d \tau
$$

Then separate variables in equation (1) and use the boundary conditions (4)-(6), we the equation

$$
\bar{\theta}(r, z, s)=\sum_{n=1}^{\infty} \bar{C}_{n}\left(\lambda_{n}, s\right) \exp \left(-z \sqrt{\lambda_{n}^{2}+s / a}\right) J_{0}\left(\lambda_{n} \rho\right)
$$

where, $\bar{C}_{n}\left(\lambda_{n}, s\right)$ are unknown coefficients, $\lambda_{n}$ is the root of Bessel function of the first kind and order zero; $J_{0}\left(\lambda_{n} \rho\right)$. Moreover, $\alpha=R / r_{0}, \rho=r / r_{0}$; dimensionless variables, $s$ is the parameter of L-transform.

Now by using the mixed conditions (2) and (3) applied to (7), we obtain a DSE to determine the unknown coefficients $\bar{C}_{n}\left(\lambda_{n}, s\right)$

$$
\begin{gathered}
\sum_{n=1}^{\infty} \bar{C}_{n}\left(\lambda_{n}, s\right) \sqrt{\lambda_{n}^{2}+s / a} J_{0}\left(\lambda_{n} \rho\right)=\bar{f}_{1}(\rho, s) ; \quad 0 \leq \rho<1 \\
\sum_{n=1}^{\infty} \bar{C}_{n}\left(\lambda_{n}, s\right) J_{0}\left(\lambda_{n} \rho\right)=\bar{f}_{2}(\rho, s) ; \quad 1<\rho<\alpha
\end{gathered}
$$

as $s \rightarrow 0$ the DSEs (8), (9) were introduced to the stationary solution of the DSE involving Laplace equation with mixed conditions [7], [14].

Now to solve DSE (8), (9), we consider the substitution

$$
\bar{A}_{n}\left(\lambda_{n}, s\right)=\bar{C}_{n}\left(\lambda_{n}, s\right) \sqrt{\lambda_{n}^{2}+s / a}
$$

Thus, DSE (8), (9) will be written as:

$$
\sum_{n=1}^{\infty} \bar{A}_{n}\left(\lambda_{n}, s\right) J_{0}\left(\lambda_{n} \rho\right)=\bar{f}_{1}(\rho, s) ; \quad 0 \leq \rho<1
$$




$$
\sum_{n=1}^{\infty} \bar{A}_{n}\left(\lambda_{n}, s\right) J_{0}\left(\lambda_{n} \rho\right) / \sqrt{\lambda_{n}^{2}+s / a}=\bar{f}_{2}(\rho, s) ; \quad 1<\rho<\alpha
$$

Then, applying the inversion Fourier-Bessel transform formula for (10) to have [4], [3]

$$
\bar{A}_{n}\left(\lambda_{n}, s\right)=\frac{2}{\alpha^{2} J_{1}^{2}\left(\lambda_{n} \alpha\right)}\left\{\int_{0}^{1} \bar{f}_{1}(u, s) J_{0}\left(\lambda_{n} u\right) u d u+\int_{1}^{\alpha} \bar{h}(u, s) J_{0}\left(\lambda_{n} u\right) u d u\right\} .
$$

Such that $\bar{h}(u, s)$ is unknown function defined over the interval $(1, \alpha), h(u, \tau)=L^{-1}[\bar{h}(u, s)]$. Next substitute (12) into (11), we obtain a first kind Fredholm integral equation of the form

$$
\int_{1}^{\alpha} \bar{K}(u, \rho, s) \bar{h}(u, s) d u=\bar{F}_{1}(\rho, s)
$$

where

$$
\begin{gathered}
\bar{K}(u, \rho, s)=\frac{2}{\alpha^{2}} \sum_{n=1}^{\infty} \frac{J_{0}\left(\lambda_{n} \alpha\right) J_{0}\left(\lambda_{n} u\right)}{\sqrt{\lambda_{n}^{2}+s / a} J_{1}^{2}\left(\lambda_{n} \alpha\right)} \\
\bar{F}_{1}(\rho, s)=\bar{f}_{2}(\rho, s)-\int_{0}^{1} \bar{K}(u, \rho, s) \bar{f}_{1}(u, s) d u .
\end{gathered}
$$

The inverse L-transform for (13) exists

$$
\int_{0}^{\tau} \int_{1}^{\alpha} K(u, \rho, t-\xi) h(u, \xi) d u d \xi=F_{1}(\rho, \tau)
$$

where

$$
\begin{gathered}
K(u, \rho, t-\xi)=\frac{2}{\alpha^{2}} \sqrt{\frac{a}{\pi}} \sum_{n=1}^{\infty} \frac{J_{0}\left(\lambda_{n} \rho\right) J_{0}\left(\lambda_{n} u\right)}{J_{1}^{2}\left(\lambda_{n} \alpha\right)} \frac{\exp \left[-\lambda_{n}^{2} a(t-\xi)\right]}{\sqrt{t-\xi}} \\
F_{1}(\rho, \tau)=f_{2}(\rho, \tau)-\int_{0}^{\tau} \int_{0}^{1} K(u, \rho, t-\xi) f_{1}(\rho, \xi) d u d \xi
\end{gathered}
$$

where Schiff [13]

$$
L^{-1}\left[\sqrt{\lambda_{n}^{2}+s / a}\right]=\sqrt{\frac{a}{\pi}} \frac{\exp \left[-\lambda_{n}^{2} a \tau\right]}{\sqrt{\tau}} .
$$

The second set of a mixed boundary conditions on a surface cylinder $z=0$;

$0<r<r_{0}<R$

$$
\begin{gathered}
\theta(r, 0, \tau)=f_{1}(r, \tau) ; 0 \leq r<r_{0} \\
\theta_{z}(r, 0, \tau)=f_{2}(r, \tau) ; r_{0}<r<R
\end{gathered}
$$

In similar manner, separate variables in (1), then use the mixed conditions (15),(16) in L-transform, we obtain a DSE for determination $\bar{C}_{n}\left(\lambda_{n}, s\right)$

$$
\begin{gathered}
\sum_{n=1}^{\infty} \bar{C}_{n}\left(\lambda_{n}, s\right) J_{0}\left(\lambda_{n} \rho\right)=\bar{f}_{1}(\rho, s) ; 0<\rho<1 \\
\sum_{n=1}^{\infty} \bar{C}_{n}\left(\lambda_{n}, s\right) \sqrt{\lambda_{n}^{2}+s / a} J_{0}\left(\lambda_{n} \rho\right)=\bar{f}_{2}(\rho, s) ; 1<\rho<\alpha
\end{gathered}
$$


Next, use the substitution $\bar{A}_{n}\left(\lambda_{n}, s\right)=\bar{C}_{n}\left(\lambda_{n}, s\right) \sqrt{\lambda_{n}^{2}+s / a}$, then apply the inversion formula in (18) we have

$$
\bar{A}_{n}\left(\lambda_{n}, s\right)=\frac{2}{\alpha^{2} J_{1}^{2}\left(\lambda_{n} \alpha\right)}\left(\int_{0}^{1} \bar{g}(u, s) J_{0}\left(\lambda_{n} u\right) u d u+\int_{1}^{\alpha} \bar{f}_{2}(u, s) J_{0}\left(\lambda_{n} u\right) u d u\right)
$$

where $\bar{g}(u, s)$ is unknown function defined over the interval $(0,1)$. A Fredholm integral equation of the first kind is obtained to determine the unknown $\bar{g}(u, s)$

$$
\int_{0}^{1} \bar{K}(u, \rho, s) \bar{g}(u, s) d u=\bar{F}_{2}(\rho, s) ; \quad 0<\rho<1
$$

. With kernel and free term respectively

$$
\begin{gathered}
\bar{K}(u, \rho, s)=\frac{2}{\alpha^{2}} \sum_{n=1}^{\infty} \frac{J_{0}\left(\lambda_{n} \alpha\right) J_{0}\left(\lambda_{n} u\right)}{\sqrt{\lambda_{n}^{2}+s / a} J_{1}^{2}\left(\lambda_{n} \alpha\right)}, \\
\bar{F}_{2}(\rho, s)=\bar{f}_{1}(\rho, s)-\int_{1}^{\alpha} \bar{f}_{2}(u, \rho, s) \bar{K}(\rho, u, s) d u
\end{gathered}
$$

where $\bar{K}(u, \rho, s)$ is the same value in (13), and the inverse L-transform is always exists.

\section{Solution integral equation (13)}

In this section, we will use the regularization method for solving the resulting Fredholm integral equation of the previous section. Regularization method is an effect tool for solving a first kind Fredholm integral equations [10, 18,2,12, 17,1]. Firstly, integral equation (13) can be written as

$$
\gamma \bar{h}_{\gamma}(\rho, s)=\bar{F}_{2}(\rho, s)-\int_{1}^{\alpha} \bar{K}(u, \rho, s) \bar{h}_{\gamma}(u, s) d u
$$

$\gamma$ is a small positive parameter, rewrite (20) as

$$
\bar{h}_{\gamma}(\rho, s)=\frac{1}{\gamma} \bar{F}_{2}(\rho, s)-\frac{1}{\gamma} \int_{1}^{\alpha} \bar{K}(u, \rho, s) \bar{h}_{\gamma}(u, s) d u
$$

The solution $\bar{h}_{\gamma}(u, s)$ converges to $\bar{h}(u, s)$ as $\gamma \rightarrow 0$. Adomian decomposition iteration is applicable for solving (20), however for simplification (20) without loss of generality, we consider

$$
\begin{gathered}
\bar{f}_{2}(\rho, s)=0, \bar{f}_{1}(\rho, s)=F(\rho, s), \\
\overline{\tilde{K}}(u, \rho, s)=\frac{2}{\alpha^{2}} \sum_{n=1}^{N} \frac{J_{0}\left(\lambda_{n} \alpha\right) J_{0}\left(\lambda_{n} u\right)}{\sqrt{\lambda_{n}^{2}+s / a} J_{1}^{2}\left(\lambda_{n} \alpha\right)}+E_{N+1}
\end{gathered}
$$

$E_{N+1}$ is an error

$$
E_{N+1}=\frac{2}{\alpha^{2}} \sum_{n=N+1}^{\infty} \frac{J_{0}\left(\lambda_{n} \alpha\right) J_{0}\left(\lambda_{n} u\right)}{\sqrt{\lambda_{n}^{2}+s / a} J_{1}^{2}}\left(\lambda_{n} \alpha\right)
$$

(20) by meaning (21) becomes

$$
\overline{\tilde{h}}_{\gamma}(\rho, s)=\frac{1}{\gamma} \bar{F}(\rho, s)-\frac{1}{\gamma} \int_{1}^{\alpha} \overline{\tilde{K}}(u, \rho, s) \overline{\tilde{h}}_{\gamma}(u, s) d u
$$


$\overline{\tilde{h}}_{\gamma}(\rho, s)$ is the approximation solution of $\bar{h}_{\gamma}(\rho, s)$. Integral equation (22) can be solved by successive approximation

$$
\begin{gathered}
\overline{\tilde{h}}_{\gamma(m+1)}(\rho, s)=\frac{1}{\gamma} \bar{F}(\rho, s)-\frac{1}{\gamma} \int_{1}^{\alpha} \overline{\tilde{K}}(u, \rho, s) \overline{\tilde{h}}_{\gamma m}(u, s) d u \\
\overline{\tilde{h}}_{\gamma 1}(\rho, s)=\frac{1}{\gamma} \bar{F}(\rho, s), \overline{\tilde{h}}_{m}(u, s)=\lim _{\gamma \rightarrow 0} \overline{\tilde{h}}_{\gamma m}(u, s)
\end{gathered}
$$

Use the summation of monograph [18], where $\overline{\tilde{h}}_{\gamma}(\rho, s)=\sum_{m=0}^{\infty} \overline{\tilde{h}}_{\gamma m}(u, s)$. It is interesting to note that, the regularization method is not commonly used for solving Fredholm integral equations of first kind. Where, some numerical techniques could be used for solving the integral equations [17], [11].

\section{Conclusion}

In this article we extend the use of the DSE method to solve nonstationary heat equation with mixed boundary conditions of the first and of the second kind, solution of the given problem is introduced to a Fredholm integral equation of the first kind, which is treated by regularization technique. This technique will be used to solve several problems dealing with mixed problems with different coordinate systems and various physical and technical sciences.

\section{Competing interests}

The authors declare that they have no competing interests.

\section{Authors' contributions}

All authors have contributed to all parts of the article. All authors read and approved the final manuscript.

\section{References}

[1] Ahmet Alturk. The regularization-homotopy method for the two-dimensional fredholm integral equations of the first kind. Mathematical and Computational Applications, 21(2):9, 2016.

[2] HO Bakodah, M Al-Mazmumy, and SO Almuhalbedi. Application of restarted adomian decomposition method on nonlinear system of integro-differential equations.

[3] Naser A Hoshan. The dual integral equations method for solving hlmholtz mixed boundary value problem. American J. of computational and applied mathematics, 3(2):138-142, 2013.

[4] Naser A Hoshan. The dual integral equations method involving heat equation with mixed boundary conditions. Engineering Mathematics Letters, 2(1):137-142, 2013.

[5] Naser A Hoshan. Solution of Fredholm integral equations of the first kind. Applied mathematical sciences, 7(77):3847-3852, 2013.

[6] Naser A Hoshan. Dual series method for solving helmholtz equation with mixed boundary conditions of the third kind. International Journal of Applied Mathematics Research, 3(4):473, 2014.

[7] Naser A Hoshan and Yousef Al-Jarrah. Dual series method for solving heat equation with mixed boundary conditions. Int. J. Open Problems Compt. Math., 7(2):62-72, 2014.

[8] Naser A Hoshan and Yousef Al-Jarrah. Cosine integral transform for solving helmholtz equation with mixed boundary conditions. Far East J. of Math. Sci., 102(1):235-247, 2017.

[9] Nasser A Hoshan. The dual integral equation method for solving the heat conduction equation for an unbounded plate. Computational Mathematics and Modeling, 21(2):226-238, 2010. 
[10] Ibrahim Issaka, William Obeng-Denteh, Isaac Owusu Mensah, Edward Prempeh, and Patrick Akwasi Anamuah Mensah. On the regularization-homotopy analysis method for linear and nonlinear fredholm integral equations of the first kind. 2017.

[11] En-Bing Lin and Yousef Al-Jarrah. A wavelet based method for the solution of fredholm integral equations. American Journal of Computational Mathematics, 2(02):114, 2012.

[12] Ahmad Molabahrami. An algorithm based on the regularization and integral mean value methods for the fredholm integral equations of the first kind. Applied Mathematical Modelling, 37(23):9634-9642, 2013.

[13] Joel L Schiff. The Laplace transform: theory and applications. Springer Science \& Business Media, 2013.

[14] Ian Naismith Sneddon. Mixed boundary value problems in potential theory. 1966.

[15] Yos Sompornjaroensuk and Kraiwood Kiattikomol. Dual-series equations formulation for static deformation of plates with a partial internal line support. Theoretical and Applied Mechanics, 34:221-248, 2007.

[16] Ya So Uflyand. Dual integral equations method in problems of mathematical physics, 1977.

[17] Abdul-Majid Wazwaz. The regularization method for fredholm integral equations of the first kind. Computers \& Mathematics with Applications, 61(10):2981-2986, 2011.

[18] F Ziyaee and A Tari. Regularization method for the two-dimensional fredholm integral equations of the first kind. Int. J. Nonlinear Sci, 18:189-194, 2014. 